\title{
La Radio italienne et la didactique du FLE dans les années 1949-1974: esquisse d'un parcours de recherche
}

\section{Rachele Raus}

\section{OpenEdition}

Édition électronique

URL : https://journals.openedition.org/dhfles/1266

DOI : $10.4000 /$ dhfles. 1266

ISSN : 2221-4038

Éditeur

Société Internationale pour l'Histoire du Français Langue Étrangère ou Seconde

Édition imprimée

Date de publication : 1 juin 2004

Pagination : 121-143

ISSN : 0992-7654

Référence électronique

Rachele Raus, « La Radio italienne et la didactique du FLE dans les années 1949-1974 : esquisse d'un parcours de recherche ", Documents pour l'histoire du français langue étrangère ou seconde [En ligne], 32 | 2004, mis en ligne le 15 décembre 2011, consulté le 27 mai 2021. URL : http:// journals.openedition.org/dhfles/1266 ; DOI : https://doi.org/10.4000/dhfles.1266

Ce document a été généré automatiquement le 27 mai 2021.

(c) SIHFLES 


\title{
La Radio italienne et la didactique du FLE dans les années 1949-1974: esquisse d'un parcours de recherche
}

\author{
Rachele Raus
}

\section{Préliminaires}

1 Cette recherche s'est heurtée à un ensemble de difficultés pour plusieurs raisons. D'abord, «La radio est [...] un monde souterrain, peu connu, fait d'interstices, éphémère, destiné à se perdre en tant que voix qu'aucune loi ni usage n'a suggéré d'enregistrer" (Menduini 2001: 8)1. Ensuite, la Radiotelevisione Italiana (RAI), qui possède les archives des enregistrements radiophoniques, ne permet pas aux chercheurs d'en consulter directement le catalogue.

D'une recherche demandée au personnel de Rome, il est ressorti que les premiers enregistrements disponibles datent de 1971. Le catalogue de Turin, ville qui, jusqu'en 1951, était avec Rome le centre des émissions de la RAI, a donné des résultats similaires, les premiers enregistrements datant de $1974^{2}$. Ces résultats nous ont étonnée, dans la mesure où nous avions précédemment trouvé à la Bibliothèque Nationale de Turin les livres qui, à partir de 1949, accompagnent les cours radiophoniques de FLE. Tout en ayant conscience que notre recherche présenterait des lacunes, nous avons quand même analysé les documents sur papier et les enregistrements disponibles.

Nous donnerons un court aperçu historique de la radio italienne $(\S 1)$, ensuite nous introduirons des réflexions sur la radio en tant qu'instrument didactique (\$2). Nous passerons à l'analyse du corpus ( $\$ 3$ ), qui se compose des livres relatifs aux cours de 1949 et de 1952 de Gilbert Varal (\$4), de ceux de 1959 et de 1973 d’Enrico Arcaini (§ 5-6), et de l'émission radiophonique relative au cours d'Arcaini de 1974 (§7). Finalement nous en tirerons des réflexions générales. 


\section{La radio dans l'histoire : quelques repères}

4 La Radio italienne commence son activité en 1924, date qui marque aussi la naissance de l'URI (Unione Radiofonica Italiana). Pendant les années 1920, le ministre Ciano préfère assurer le contrôle italien des moyens de communication et favorise donc des entrepreneurs italiens. C'est le début du lien entre économie et pouvoir politique, qui aboutira au contrôle étatique de la période fasciste. En 1924 la radio passe de la musique classique, des bulletins d'informations et des entretiens. On s'adresse surtout à un public de jeunes amateurs : l'écoute se fait à la maison, dans un espace privé. L'URI essaie de promouvoir l'écoute de la radio pour produire une consommation de masse, mais elle se heurte à des problèmes concrets, comme l'analphabétisme et la présence remarquable de dialectes.

5 En 1925 apparaissent les premiers référendums pour connaître les préférences du public. Le résultat du questionnaire de 1927 montre que le public demande « des cours de radiotechnique, des transmissions en prose, des cours de langues étrangères " (Monteleone 1995: 39). A la suite de ces sondages, quelques années plus tard, le fascisme inaugure la «Radio per le Scuole» (Radio pour les écoles), ce qui ne veut pas seulement dire la création de programmes conçus à des fins didactiques, mais qui renvoie également à l'installation d'appareils radiophoniques dans les écoles.

En 1928 l'URI devient l'EIAR (Ente Italiano per le audizioni radiofoniche) qui favorise la création d'un réseau national, mais le coût du «canone $»^{3}$ est excessif, ce qui fait que le public reste essentiellement bourgeois. Malgré les efforts du régime fasciste pour créer un public de masse, la radio reste donc un symbole de statut social. Outre les enfants, ce sont les femmes qui écoutent la radio beaucoup plus que les hommes. Le public commence à présenter un certain profil, ce qui suppose que la radio a désormais trouvé une place légitime à l'intérieur des maisons.

7 Pendant les années 1935-45 le régime fasciste utilise la radio pour sa propagande. De 2718 postes d'écoute et haut-parleurs dans les écoles primaires en 1934, on passe, quatre ans après, à 23945 postes $^{4}$. L'écoute radiophonique se fait de plus en plus publique : on écoute la radio à l'école, mais également sur les places des villes, où le régime se hâte d'installer des haut-parleurs, et dans les locaux du «dopolavoro »".

8 En 1944 l'EIAR devient la RAI (Radio Audizioni Italia), gérée par l'État, même si le Parlement essayera d'assurer la démocratie radiophonique, par la création, en 1947, d'une Commission parlementaire de vigilance sur l'indépendance politique et l'objectivité informative à la radio. A partir de 1944 on organise une base d'émission à Turin, et une autre à Rome, jusqu'en 1951, quand la RAI ne garde que Rome comme base d'émission. On structure les émissions en trois chaînes et on organise un véritable palimpseste, c'est-à-dire une programmation précise des émissions pendant la journée. La première chaîne a des caractéristiques généralistes ; la deuxième se veut essentiellement de divertissement; la troisième est culturelle. La radio doit en outre commencer à se réorganiser à cause de la concurrence de la télévision qui, à partir des années 1950, entre dans les maisons des Italiens. On parle, en effet, d'une crise de la radio : l'écoute de la radio devient de plus en plus une écoute de fond pour se transformer en un bruit qu'on préfère éviter, les gens optant pour les « temps dilatés » (Monteleone 1995 : 270) de la télévision.

Pour ce qui est des langues : 
« Les programmes de La radio pour les écoles débutèrent en 1947 et visaient à intégrer les connaissances linguistiques et les curiosités culturelles d'une population scolaire souvent dans l'impossibilité économique d'acheter les livres scolaires. Ces programmes étaient diversifiés par rapport aux élèves du primaire et à ceux des collèges. Les émissions étaient limitées aux horaires du matin (entre 10h et 11h30) sur une durée d'une demi-heure, les jours ouvrables, selon le calendrier scolaire d'octobre à juin. La radio pour les écoles était conçue pour intervenir pendant le cours non pas en tant que simple instrument récréatif, mais en tant que substitut temporaire de l'enseignant. » (Monteleone 1995 : 251-252).

10 Le 10 avril 1954 la RAI se renomme en Radiotelevisione Italiana, nom qu'elle a encore aujourd'hui. Le changement est dû à la présence de la télévision, dont l'utilisation devient de plus en plus fréquente, grâce à l'invention des transistors qui remplacent les lampes, produisant une baisse remarquable des prix. Les années 1960 se caractérisent par le succès de la télévision, qui produit d'autres changements dans l'utilisation de la radio. L'écoute de cette dernière devient de plus en plus individuelle et suppose un public composé d'auditeurs qui sont avant tout des téléspectateurs. Les années 1970 ne marquent pas de gros changements si ce n'est la diffusion massive des médias : c'est l'aube de la culture médiatique telle que nous la connaissons encore aujourd'hui. Après ce parcours historique, demandons-nous à présent quel a été le rôle de la radio dans la didactique des langues étrangères.

\section{La radio et la didactique des langues étrangères}

11 Fenati et Scaglioni parlent des "potentialités éducatives de la radio pour l'alphabétisation et plus généralement pour élever le niveau culturel sur le modèle de l'élite socioculturelle » (2002:7).

12 La radio en Italie avait en effet trois objectifs : « informer, éduquer, amuser » (ibidem : 8), ce qui se concrétisera dans la programmation sur trois chaînes. Pour ce qui est de la didactique, on utilisait «la radio et la télé pour remédier au manque d'écoles et d'assiduité scolaire pour les enfants de 11 à 14 ans » (Monteleone 1995 : 309).

D'ailleurs, pendant les années 1950 on assiste à une attention particulière portée à la radio en tant qu'instrument de formation, au point qu'à Paris paraît le rapport de l'Unesco sur l'organisation de la radio pour l'école. Au-delà des écoles, «l'action de vulgarisation culturelle fut conduite par la radio sous plusieurs formes : une offre consistante de cours de langues étrangères et des sections informatives de plusieurs genres » (ibidem : 256).

14 Ce sont les livres correspondant à ces émissions à partir de 1949 que nous avons retrouvés.

15 Avant d'entrer en détail dans l'analyse du corpus, il nous est utile de voir quelques éléments caractérisant le support radiophonique en relation avec l'aspect didactique. La caractéristique essentielle de la radio est sans doute l'oralité, mais à la radio, l'oral n'est pas un oral spontané, c'est un oral qui «se fait écouter» (Hendy 2002 : 205) : les dialogues visent le réalisme, mais ne sont en fait que le produit de la stratégie didactique.

Ensuite, il faut souligner que l'élément auditif stimule l'imagination, la radio permettant une construction du sens et des images que la télévision ne permet pas, cette dernière produisant une restriction de l'imaginaire collectif ${ }^{6}$. Ce qui est en jeu 
dans le dualisme radio/télévision, c'est le pouvoir de la langue, qui évoque des images mentales du monde, contre le pouvoir de l'image en soi. En ce sens, le fait de décrire la télévision comme une "radio avec des images ", comme le fait David Morley ${ }^{7}$ nous semble faux, dans la mesure où les images donnent au son de la télévision un statut différent de celui de la radio.

Un autre élément à souligner, c'est que la radio permet au médium de devenir le message, selon l'expression de McLuhan. Non seulement l'écoute nous permet de créer des images sur le contenu de ce qui est dit, mais c'est également le signifiant qui contribue à évoquer des mondes, si nous considérons le signifiant à la façon saussurienne comme image acoustique. Le cas de la langue étrangère est révélateur, parce que l'auditeur s'attarde sur le signifiant des mots, les ressentant comme appartenant à un pays étranger avec tout ce que cela apporte par association. En ce sens, la radio produit une modalité d'écoute qui se veut en même temps informative et associative ${ }^{8}$. Ce n'est pourtant que vers la fin des années 1950 que l'on prend conscience des possibilités du support radiophonique. Au début, les efforts des éducateurs sont plutôt centrés sur la légitimation de l'appareil radiophonique à des fins didactiques, comme le démontre le premier dialogue du cours de 1949, tenu par Gilbert Varal :

«Paul - Qu'attends-tu à la radio ?

Jean - J'attends la leçon d'anglais.

Paul - Aurais-tu donc l'intention d'apprendre la langue anglaise par la radio?

Jean - Ça te surprend tellement, mon vieux? Pense qu'il y a déjà bien des années que dans les programmes d'émission des principaux pays du monde on trouve des cours réguliers de langues étrangères!

Paul - Mais, alors, Jean, sincèrement, les crois-tu utiles ?

Jean - Mais bien sûr, mon ami : il suffit d'être attentif, et de suivre avec un peu de bonne volonté les conseils du professeur » (1949 : 5-6).

Un autre dialogue nous permet d'autres observations :

«Paul - Et ton poste [il s'agit du poste de radio], fonctionne-t-il bien?

Jean - Juge par toi-même, j'en suis très content.

Paul - Il a dû coûter cher, je pense.

Jean - Pas du tout, mon vieux, je l'ai payé comptant et j'ai obtenu une réduction de

$10 \%$ et de cette façon il ne m'a coûté que 9000 francs [...]

Paul - J'ai bien envie d'en acheter un et j'espère pouvoir le faire cette année "

(ibidem : 8).

19 Ce qui est clair ici, c'est la promotion de l'objet, dont le prix commence à baisser en 1949, permettant à la radio de devenir un moyen de communication de masse. En ce sens, considérant les abonnements à la radio, on en compte 40778 en 1927 contre 2566258 en 1949 (Menduini 2001: 235), et le chiffre augmentera jusqu'en 1958, pour ensuite décroître en raison du succès de la télévision. Ces formes de légitimation et de promotion disparaissent dans le cours d'Arcaini de 1959.

Un autre point à considérer, c'est qu'à partir de 1949 les cours à la radio s'accompagnent de livres. Nous lisons dans la préface du cours de Varal de 1949 que ces cours se tenaient à la radio depuis un certain temps. Pourtant, c'est à partir de 1949 qu'on ressent l'exigence de créer une sorte de paquet multimédial ${ }^{9}$ : le cours radiophonique s'accompagnera d'un livre. Varal en donne la raison suivante :

"plusieurs professeurs et spécialistes en langues étrangères ont affirmé que par l'utilisation d'un texte, accompagnant l'écoute de courtes leçons à la radio, l'étude d'une langue étrangère à travers la radio est de beaucoup plus efficace [...]: l'élément phonique et l'élément auditif sortent enrichis, pour ainsi dire, de l'élément visuel. L'auditeur... remarquera tout de suite les progrès faits, qui 
rapprochent de beaucoup l'enseignement par radio de l'enseignement ancien et

traditionnel de la classe scolaire » (ibidem : 3 ).

21 Ces « courtes leçons à la radio » duraient un quart d'heure-vingt minutes. D'après la préface, Varal opère un choix dû à la volonté de rapprocher la didactique radiophonique de l'approche traditionnelle de la classe, ce qui contribuera ultérieurement à la légitimation de la méthode radiophonique.

Une autre considération à faire est que la radio avait été pendant longtemps une "traductrice » de culture, dans le sens où elle traduisait l'écriture en voix et son ${ }^{10}$. Le fait alors de ressentir l'exigence, à partir des années 1950, d'intégrer à l'émission radiophonique un texte écrit en tant qu'élément visuel, nous pose la question de savoir si la multimodalité de la télévision n'est pas ressentie comme une menace, vu la monomodalité de l'écoute radiophonique. Quoi qu'il en soit, ce qui est certain, c'est que, tout comme dans la méthode audio-visuelle (MAV) élaborée à partir des années 1950, il y a une étroite association entre l'image et le son à des fins didactiques, au point que, comme le précise Varal, il faut considérer le livre et les émissions comme s'intégrant mutuellement.

\section{Le profil de l'apprenant/lecteur-auditeur}

Pour ce qui est de l'image de l'auditeur inscrite dans le texte, Varal, dans sa préface de 1949, parle d'un auditeur «qui est animé de bonne volonté et qui est décidé à apprendre ». Ceci nous montre également combien la méthode orale tout comme la méthode audio-visuelle, étaient centrées sur un savoir que l'apprenant ne devait acquérir que patiemment. Cependant cet apprentissage à distance suppose, dans la triade apprenant-objet-enseignant, un rôle plus actif de l'apprenant par rapport à la méthode traditionnelle en classe. Toujours dans Varal, nous lisons :

«Aux auditeurs les plus appliqués, nous donnons donc la possibilité de faire des exercices et de les corriger tout seuls, en s'aidant par le texte lui-même : en effet, dans ce texte il y a tout ce qu'il faut pour l'autocorrection [...] sans doute, il faut ne pas se contenter d'écouter les courtes leçons à la radio, encore faut-il y ajouter le plus souvent possible les émissions normales de l'oral du pays dont on étudie la langue ; lire les journaux, qui eux aussi utilisent une langue vive et actuelle... » (1949: 3-4).

24 L'invitation de Varal à suivre les émissions radiophoniques en langue étrangère, pour entendre la façon de parler du pays, est due au fait que ces émissions étaient déjà fréquentes pendant la période fasciste, bien que le régime en ait interdit l'écoute en raison d'un contenu souvent révolutionnaire (par exemple, Radio Belgrade).

Dans un autre passage de Varal (p. 81), nous trouvons des éléments complémentaires concernant cet apprenant qu'il appelle lecteur-auditeur. Dans la leçon LII, il y a la lettre suivante :

«Monsieur le professeur,

je me permets de vous adresser cette lettre pour vous dire que j'écoute avec beaucoup d'attention les leçons de français à la radio et j'espère qu'elles me seront utiles pour mon travail. Depuis quelque temps, en effet, je suis employée comme dactylographe dans une grande maison de commerce qui exporte des marchandises surtout vers la France; par conséquent je dois écrire à la machine beaucoup de lettres en français et mes supérieurs voudraient me dicter le texte au lieu d'en écrire le brouillon pour faire plus vite; mais cela est impossible parce que je fais beaucoup de fautes... de frappe. Je vous remercie donc dès maintenant pour tout ce 
que vous nous apprendrez par rapport à l'exacte compréhension de ce qu'on écoute " (ibidem : 81-82). radiophonique du cours de 1974, grosso modo une réplique des cours de 1971 et de 1973, Arcaini précise que son cours s'adresse à tous, et en particulier à ceux qui sont intéressés par la langue en tant qu'instrument de culture et de communication. L'optique va sans doute dans le sens d'une approche communicative et en tout cas d'une utilisation de masse de la radio.

\section{Les cours de Gilbert Varal de 1949 et de 1952}

31 Dans le cours de Varal de 1949, les leçons s'ouvrent avec une partie théorique qu'accompagne l'écoute radiophonique. L'écriture est conçue pour la radio comme le montrent les mécanismes phatiques d'appel à l'auditoire («Maintenant écoutez tout l'alphabet... comme vous avez pu entendre »). Les leçons I-VI sont dédiées à l'alphabet et à la phonétique et présentent des dialogues entre Paul et Jean; à partir de la leçon VII, on étudie la grammaire et le vocabulaire, préférant au dialogue des textes de civilisation, littérature et actualité. Au début de la $8^{\mathrm{e}}$ leçon, Varal précise que « Nous 
devons connaître le pays dont nous étudions la langue: voilà pourquoi ces courtes leçons auront pour base un texte instructif simple, à partir duquel nous ferons des observations de grammaire et de langue » $(1949: 15)$.

On remarquera la présence d'un premier nous qui au lieu d'être inclusif vise la tournure impersonnelle à valeur déontologique (nous devons connaitre le pays = il faut $/$ il faudrait connaître), et ensuite d'un nous de majesté (nous ferons remarquer = moi en tant que professeur). Dans les deux cas, il s'agit de poser l'objet / apprentissage de la langue comme allant du haut vers le bas, approche caractérisant toute méthode d'apprentissage des années 1950. La présence de plusieurs renvois d'une leçon à l'autre montre que le cours demande une écoute assidue de l'auditeur. Le renvoi du texte italien à sa version française et vice-versa permet l'autocorrection des traductions, d'autres renvois sont riches en renseignements, comme celui-ci : «Nous avons étudié dans notre $9^{e}$ leçon les frontières de la France et nous avons dit un mot sur sa géographie physique ; disons ce soir quelque chose sur sa géographie économique » (p. 32). L'indication temporelle place l'émission dans la soirée ou tard dans l'après-midi. Pour ce qui est des pronoms, outre les nous de majesté (nous avons dit un mot... disons ce soir), il y a aussi la présence de deux nous inclusifs (nous avons étudié dans notre $9^{\mathrm{e}}$ leçon), ce qui en quelque sorte rapproche l'énonciateur de l'auditoire. En ce sens, l'écoute favorise le lien communautaire non seulement entre auditeurs mais également entre l'auditoire et l'enseignant. Hendy souligne que l'écoute favorise les relations humaines (2002: 157), ce qui est encore plus évident à la radio parce que la présence simultanée de l'émetteur et des auditeurs renforce la sensation d'appartenance communautaire, créant une sorte de classe virtuelle.

Deux éléments montrent la présence sous-jacente de la méthode audio-orale (MAO) : la phraséologie et la présence de scripts, c'est-à-dire de situations stéréotypées. La phraséologie apparait à partir de la leçon XII pour donner des expressions courantes de la vie quotidienne et pour la correspondance commerciale et d'affaires, ce qui confirme l'ethos de la secrétaire comme l'un des profils idéaux. Pour ce qui est des scripts, il y a deux situations où ils sont tout particulièrement présents: le dialogue à la banque (leçon XXVII) et celui à l'hôtel (leçon XXXVII).

Disons pour résumer que le cours de Varal se caractérise par la co-présence de la méthode traditionnelle (Martinez 1996 : 49-51), dite aussi de la grammaire-traduction, d'un côté, et de l'approche audio-orale, ici partiellement contrastive, de l'autre. Pour ce qui est de la première, en voici les caractéristiques : un apprentissage centré sur le vocabulaire et la grammaire comme objectifs immédiats ; la présence de règles prescriptives et d'un système linguistique dont les modèles sont en grande partie les bons auteurs qu'il faut traduire. L'approche audio-orale intègre la précédente dans la mesure où elle vise une compétence orale qui, comme dans la lettre de la dactylographe pour la compréhension orale, se fait nécessaire. Pourtant, dans le cours de Varal, la méthode traditionnelle l'emporte, l'auteur insistant fortement sur la compétence d'écriture à côté d'une compétence orale portée essentiellement par la radio.

Le cours de 1952 se veut une simple mise à jour du précédent. Dans la préface du livre, nous lisons :

«Pour répondre aux requêtes de plusieurs auditeurs, cette réédition a été augmentée par l'ajout des auxiliaires, des quatre conjugaisons des verbes réguliers et des irréguliers les plus importants, et également d'autres exercices de traduction, de dialogues divers, d'un vaste recueil de phrases idiomatiques d'usage courant et des notes ajoutées à quelques leçons. » 
tructure du cours se modifie en acquérant de plus en plus les modalités de l'approche audio-orale. Les verbes sont présentés dans des tableaux de conjugaison mis dans une section qui précède les véritables leçons et qui contient également les règles phonétiques et l'alphabet. Le lexique et la phraséologie deviennent centraux, et la première leçon, au lieu de concerner l'alphabet, fait la liste des « Phrases faciles de tous les jours ». De même, apparaissent des listes de noms de personnes et de lieux, avec leur traduction. Le changement méthodologique est évident lorsque Varal précise que : " ce livre ne veut pas être un livre de grammaire à lire au micro pour... faire fuir les auditeurs "(1952: 245). La première version présentait des parties de grammaire très denses. Au-delà d'une simple mise à jour, la version du cours de 1952 marque un pas vers la méthode audio-orale: l'approche contrastive, la phraséologie, la décontextualisation sont des éléments qui vont dans cette direction.

Nous voudrions revenir sur l'observation que l'oral à la radio n'est pas spontané, les dialogues participant à la stratégie de l'enseignant. Dans le dialogue " Au théâtre », il y a bien des passages qui visent à recréer l'image et qui appartiennent plutôt à l'écriture qu'à l'oralité, cette dernière étant sans doute plus spontanée : « Admire les toilettes des dames et leurs bijoux! Le beau coup d'œil !... Voilà le rideau qui se lève, la représentation va commencer ». L'absence d'interjections contribue à ce manque de spontanéité : "Donnez-moi donc, s.v.p., le fauteuil en question", où le donc nous semble être un connecteur plutôt fort en ce contexte, malgré l'atténuation du s.v.p. Dans l'échange chez le dentiste certains passages sont excessifs (" Hélas! L'acide hyperchloridrique qui me cause de si grands maux d'estomac, m'a gâté toutes mes dents »). De même, le dialogue "Au restaurant» témoigne d'une stratégie visant l'apprentissage du lexique (la liste des produits alimentaires) et de la phraséologie : le client se limite à dire l'essentiel, les derniers échanges sont mal liés, violant le script, par exemple dans le manque du s.v.p.

\section{Le cours d'Enrico Arcaini en 1959}

Le cours d'Arcaini de 1959 est structuré en LXXV leçons, tout comme celui de Varal. Dans la préface au cours nous lisons :

«Pour organiser ce cours pratique de LF, j'ai considéré quelques exigences de la pédagogie actuelle : - la connaissance s'inaugure dans la perception globale ; - le sujet passe donc à l'analyse pour avoir conscience des éléments qui composent ce qu'il a perçu globalement ; - le sujet reconstruit, recompose, pour créer des formes nouvelles, originales (synthèse). [...] la phrase, en tant qu'unité de pensée, est le résultat de l'élaboration produite par un «esprit " précis qui peut être très différent de l'esprit de la langue de départ. [...] Un Français ne réagit pas de la même façon qu'un Italien face au même stimulus; si parfois les mots correspondent, c'est souvent l'«accent " qui varie, en révélant ainsi un " caractère » de la langue qui représente, somme-toute, l'originalité de l'« esprit » de la langue qui se fait expression. [...] il faut lire avant le sujet du dialogue pour 'savoir au moins de quoi il s'agit'. Le travail à faire sera alors plus simple : il faudra tout simplement suivre avec attention celui qui parle. Pour provoquer les réactions automatiques dont j'ai parlé tout à l'heure, j'ai imaginé un dialogue autour d'un certain nombre de centres d'intérêt pour faire ressortir les mots ainsi que la phraséologie particulière que l'on adopte dans une situation précise, sans exagérations. » 

recourt à la pédagogie moderne pour tracer un projet didactique précis qui relève de l'approche contrastive. L'approche psychologique (perception-analyse-synthèse); le couple stimulus-réaction, ainsi que la notion de réaction automatique; la centralité du lexique, et encore plus de la phrase en tant qu'unité de base de la pensée, d'où la centralité donnée à la phraséologie; des situations précises rappelant la notion de script;l'importance du dialogue, qui l'emporte sur les traductions et les lectures. Il s'agit là d'un ensemble d'éléments qui placent la méthode d'Arcaini à l'intérieur de l'approche audio-orale, fondée sur la linguistique structurale et la psychologie béhavioriste. Pour ce qui est de la notion d' " esprit de la langue », il s'agit sans doute d'une toute première sensibilisation aux différences culturelles qui deviendront centrales à partir des années 1980 .

Par rapport aux deux cours de Varal, il y a une évolution constante d'une approche essentiellement traditionnelle vers une approche audio-orale et contrastive. Surtout, il y a toute une tradition consolidée de l'écoute radiophonique sur laquelle Arcaini s'appuie pour pousser un regard «méta » plus significatif que dans Varal. Ainsi nous trouvons une structuration précise des contenus, qui n'est jamais explicite dans Varal :

«j'ai ajouté la grammaire à la phrase grammaticale dans les leçons. On pourra l'appeler 'réflexion sur le texte, enquête sur l'expression'. [...] toute leçon s'articule de la façon suivante : 1) dialogue à trois ; 2) la phonétique ; 3) la grammaire ; 4) la phraséologie ; 5) la nomenclature ; 6) les exercices. »

Si l'on passe de la préface aux leçons, on se trouve confronté à un dialogue entre Paola et Marisa qui vont à Paris pour rendre visite à leur professeur. Les trois personnages correspondent à des personnes réelles, comme le précise Arcaini en remerciant «Mlles Marisa Barracano et Paola Musarra [...] qui ont gentiment accepté d'être les personnes principales du voyage idéal en France et qui ont contribué à créer leurs personnages ».

La présence constante des trois personnages permet d'établir un lien affectif avec l'auditoire. Ces trois personnes sont également présentes dans l'image en ouverture du texte qui évoque le scénario du dialogue. Au fond la tour Eiffel et Notre-Dame permettent d'identifier la ville de Paris, fonctionnant comme des icônèmes ${ }^{12}$. Cette nécessité de donner à voir nous fait réfléchir sur la monomodalité de la radio, ressentie comme une limite peut-être à cause de la présence de la télévision. D'ailleurs, le fait que pour les cours de FLE à la télévision on écrit aussi des textes à partir de $1959^{13}$, nous fait penser que l'élément textuel est ressenti plus généralement comme indispensable en tant qu'outil dans la didactique à distance. Le livre a en ce sens deux fonctions par rapport à l'enseignement radiophonique, d'un côté il permet de suivre en temps réel ce qui est dit à la radio et d'associer par conséquent la lecture à l'écoute orale ; de l'autre il permet de réviser en temps différé ce que l'on a fait pendant le cours, en fonctionnant alors comme un manuel traditionnel.

Dans la première leçon le rôle des trois personnages est précisé :

«Paola : [...] surtout, Marisa, c'est à toi de parler! Tu sais que je ne sais pas dire un mot en français!

Marisa : Naturellement, ne t'inquiète pas. Non seulement ce sera à moi de parler, mais je traduirai pour toi tout ce que nous dirons. "

L'auditeur peut s'identifier à Paola, Marisa acquérant plutôt le rôle d'aide-enseignant. L'identification de l'apprenant avec Paola permet à l'enseignant d'en diriger l'apprentissage. Ainsi, pour apprendre la phonétique, le mécanisme de la répétition est 
mis en scène à travers l'expédient du professeur qui invite Paola à prononcer les sons après les avoir entendus de sa bouche ou de celle de Marisa. Les mécanismes phatiques qui dans Varal s'adressaient directement à l'auditoire (" écoutez / prêts pour la dictée / ...») sont ici adressés à Paola («Alors voyons, Mlle Paola. Prononçons bien ensemble ! »).

Pour ce qui est de l'approche contrastive, il faut dire que, non seulement le dialogue se structure sur deux colonnes pour faire coexister la version italienne et sa traduction en français, mais que cette approche est présente aussi dans le dialogue et dans les tableaux. Disons, donc, que par rapport à Varal, le texte d'Arcaini représente une évolution vers la méthode audio-orale qui est clairement exposée dès la préface.

\section{Le cours d'Arcaini de 1973}

Le cours de 1973 se restructure à partir « d'idées nouvelles et d'une longue expérience radiophonique ». Arcaini abandonne la structuration en LXXV leçons, lui substituant 5 groupes de 8 émissions. Tout comme en 1959, la préface s'ouvre sur des réflexions linguistiques :

«1) la langue est un instrument de communication et doit surtout servir à parler; cela explique l'importance particulière qu'on a attribué à la langue parlée d'aujourd'hui ;

2) la langue est surtout une activité. Il s'agit d'acquérir des habitudes nouvelles [...] ;

3) la langue présentée comme modèle est celle, assez homogène, que l'on peut définir comme français standard d'aujourd'hui ;

4) la langue se constitue d'ensembles structurés [...];

5) la langue est une activité sociale. Voilà pourquoi nous avons placé les faits linguistiques dans un contexte situationnel qui permettait de récupérer la « culture » du pays, effleurant de façon sommaire les problèmes qui caractérisent les différentes couches sociales. Cela pour éviter des stéréotypes et des lieux communs qui ne décrivent pas le pays et qui ne motivent pas l'apprentissage.»

Nous trouvons la confirmation d'un décentrement culturel, l'esprit de la langue se formulant ici par la notion de "culture ». L'on commence à entrevoir le binôme cher à Galisson de la langue-culture.

Nous constatons une meilleure exploitation des éléments théoriques sur la base des expériences radiophoniques effectuées: l'abandon du dialogue entre trois personnes permet une grande souplesse des leçons. Trois éléments nous permettent de parler de méthode audio-visuelle :

1. l'abandon presque total de l'approche contrastive, ce qui, selon Martinez, caractérise l'approche audio-visuelle ;

2. l'absence totale de traductions ;

3. une grande importance donnée à l'image, qui se trouve fortement associée au texte.

49 A propos des images, rappelons avec Martinez que « On qualifiera de méthode audiovisuelle celle qui, ne s'en tenant pas seulement à associer l'image et le son à des fins didactiques, les unit étroitement, de sorte que c'est autour de cette association que se construisent les activités » $(1996: 59)$.

On peut retracer une évolution en ce sens, qui passe par les tableaux de conjugaisons du cours de Varal de 1951, par les images qui contextualisent les situations des 
dialogues dans le cours d'Arcaini de 1959, pour arriver au cours de 1973, où les images, outre pour contextualiser, sont aussi utilisées en tant que signifiantes dans les tests.

Une dernière chose est à retenir dans la préface de 1973 : « la langue de l'émission n'est pas la langue écrite, mais la langue orale. Par conséquent l'auditeur devrait au début faire abstraction du texte écrit et ne se concentrer que sur la voix. »

Si jusqu'à maintenant les éducateurs recommandaient la lecture du texte avant l'écoute radiophonique, Arcaini renverse cette séquence, recommandant de s'abstraire du texte écrit pour se concentrer sur l'écoute.

Finalement, toujours dans la préface, nous lisons :

« Nous avons voulu fournir - dans certaines limites - une image de la poésie et de la chanson française dans de 'très courts' interludes; ici encore nous avons opéré un choix précis: des poésies d'auteurs actuels, riches en contenus humains, des chansons d'une certaine valeur pour ce qui est des textes mais également pour la musique. »

On se rend compte combien l'on est loin du cours de Varal de 1949, où il n'y avait que des textes d'auteurs classiques.

Disons donc que le cours de 1973 présente la plupart des éléments qui caractérisent les méthodes audio-visuelles. En même temps, on commence à voir l'influence de l'approche communicative. Même si pour l'instant on ne tient pas compte des besoins individuels des apprenants, Arcaini parlant de l'acquisition d'une langue standard, nous pouvons remarquer des éléments qui vont dans la direction de l'approche communicative: la langue conçue comme instrument de communication nous fait penser à la volonté de donner une compétence de communication par l'acquisition de la langue; la notion de "culture " produit la contextualisation des faits linguistiques pour éviter la création d'ethno-stéréotypes.

\section{L'écoute du cours d'Arcaini de 1974}

La version radiophonique du cours Progression dont nous venons d'analyser le texte, a été consultée à la station RAI de Turin, station désormais informatique avec un ordinateur branché dans l'inter-réseau et un casque qui permet l'écoute. Dans l'interréseau de la RAI, chacune des 40 émissions a été divisée en trois-quatre éléments de grille, dont le premier et le dernier concernent les titres et l'un ou bien les deux autres sont l'émission proprement dite. Lorsqu'il y en a deux, c'est en général que le deuxième élément concerne la poésie ou la chanson émise dans la leçon. Toute leçon a une longueur de 15 minutes.

57 La première émission écoutée est une interview, où Arcaini présente le cours : il s'agit d'un cours de langue française passé à la radio en accord avec l'Ambassade de France, et à l'aide des deux collaboratrices habituelles, Marisa et Paola. Arcaini parle ensuite de la langue en tant que vision du monde faisant écho à Humboldt et confirmant sa sensibilisation à la différence culturelle. Le professeur souligne qu'« on apprend le français parce qu'il est utile et pour le plaisir ». Pour ce qui est de l'utilité du français, Arcaini parle d'une langue utile pour le commerce, soulignant que l'Italie est le deuxième partenaire officiel de la France dans le commerce, et pour le tourisme, puisque 5 millions de Français visitent notre beau pays tous les ans. L'utilité du français est également due à sa position géographique dans le monde. 
Arcaini précise le but du cours : il s'agit de permettre à l'auditeur d'acquérir les quatre compétences de base (parler, comprendre, lire, écrire), mais «sans faire de la grammaire de la langue ; plutôt il s'agit de faire de la grammaire DANS la langue ». La grammaire est alors ce regard métalinguistique sur la langue dont il était déjà question en 1959. Arcaini précise que le titre du cours qui, à partir de 1971, prend le nom de "Progression - Cours radio visuel », est dû à l'idée que la progression dans l'apprentissage d'une langue se fait «à partir des éléments acquis », et que l'écriture est étroitement liée aux illustrations annonçant les sujets. Nous retrouvons la précision qu'il faut tout d'abord écouter pour ne pas se faire influencer par le support écrit. La méthode reste la suivante :

1. 1. Écoute d'un dialogue - reprise des phrases avec les commentaires des trois personnages (traductions, éclaircissements...) - deuxième écoute pour mémoriser. Cette première séquence est suivie d'une section "Autrement dit ", où on suppose un auditeur extérieur reformulant par ses propres mots le dialogue qu'il vient d'écouter. Cette reformulation permet le passage du style direct au style indirect.

2. 2. Écoute d'une poésie ou d'une chanson contemporaine.

3. 3. Section où l'auditeur est plus « actif », en devant faire des exercices qui consistent dans la répétition et le recopiage de ce que l'on dit à la radio.

4. 4. Exercice de libre création. A partir des phrases que l'on a retenues dans la leçon, il s'agit de composer un nouveau texte pour tester la compétence acquise.

Arcaini souligne que la $8^{\mathrm{e}}$ leçon de chaque groupe d'émissions marquera un moment de vérification et de récapitulation.

60 Le premier dialogue écouté concerne un appel téléphonique. A la différence de l'appel téléphonique de Varal, où il $\mathrm{y}$ a deux personnages en présence, dans le cours d'Arcaini nous sommes pris dans un jeu de rôles : Paola assiste à l'appel de Marisa, en ne pouvant entendre que la conversation de cette dernière. L'auditeur perd donc la place privilégiée de l'« auditeur omniscient ", pour être incorporé à la situation. Ce type de sollicitation de l'auditeur est souvent accompagné d'éléments phatiques et conatifs qui invitent l'auditeur à ne pas se décourager.

61 Les dialogues manquent (toutefois) de spontanéité : la progression des tours de parole n'est pas toujours respectée et l'on s'attarde sur la prononciation. C'est le cas de l'histoire de Mme Latour (leçon 4), une dame âgée à laquelle un voleur essaie de voler son sac: le voleur accumule une série de phrases sans cohésion textuelle (« où allezvous Mme Latour?" «Ne partez pas Mme Latour ", "N'oubliez pas votre sac Mme Latour »), et sans que la dame réagisse si ce n'est tardivement par rapport à une situation réelle possible. La structure phrastique qui par les reformulations se fait de plus en plus complexe, caractérise également les phrases en « progression » :

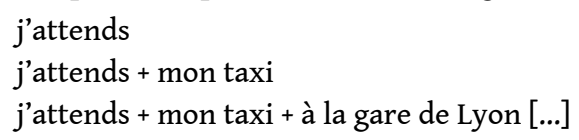

62 La $8^{e}$ émission est de récapitulation et permet de revenir sur la grammaire, puisque, comme le dit Arcaini « on fait de la grammaire APRES avoir étudié la langue ».

63 Il nous semble donc qu'en général l'écoute de quelques leçons du cours de 1974 nous permet de mieux préciser ce que nous avons considéré pour le cours de 1973. 


\section{En guise de conclusion}

L'histoire des cours de FLE à la radio correspond à l'histoire des méthodes en didactique. De la méthode traditionnelle du cours de Varal en 1949, on passe, en 1952, à une approche qui se fait de plus en plus audio-orale. Dans Arcaini 1959 la transition est faite et on entrevoit la présence de la méthode audio-visuelle, qui atteint sa réalisation complète dans le cours de 1973. Là encore, nous sommes en présence de plusieurs éléments qui vont dans la direction de l'approche communicative. Bref, une évolution qui se produit en douceur, l'approche précédente contenant déjà les présupposés de la suivante. Mais l'évolution didactique s'accompagne de la prise de conscience de l'instrument radiophonique. La radio n'est pas simplement un moyen de communication qui acquiert de l'importance à partir de la méthode audio-orale, comme on pourrait le supposer. Elle a toujours été un moyen privilégié dans l'apprentissage des langues, étant, pendant un certain temps, le seul appareil à permettre l'apprentissage à distance. Pourtant, cette relation entre radio et didactique des langues a produit très tard une réelle prise de conscience des possibilités de la radio dans l'apprentissage. Le besoin de Varal de légitimer l'appareil en tant que moyen utile et adapté à l'apprentissage à distance, témoigne du tout début de ce parcours. Les réflexions linguistiques d'Arcaini supposent déjà une familiarité avec l'appareil radiophonique dans l'activité d'apprentissage. Pourtant, la volonté d'aller dans le sens d'un paquet multimédias intégrant l'image, en accord avec les préoccupations des méthodes audio-visuelles des années 1970, nous semble vider l'appareil radiophonique de ses possibilités liées à la simple écoute, de plus en plus vécue comme une limite, et cela bien que les cours des années 1970 insistent sur le fait que l'écoute orale doit précéder la vision de l'image et la lecture. D'ailleurs, le développement par la suite des cassettes-vidéo, des supports DVD et de l'ordinateur, n'a fait que renforcer l'idée que l'écoute n'est qu'un sens fortement limité à lui tout seul. Les supports actuels, visant plutôt la multi-modalité, comme par exemple le DVD, ainsi que la "multi-médialité " (l'ordinateur est multimédial en soi, se composant d'un écran, d'un clavier, de hautparleurs...), risquent de nous faire oublier qu'il manque une réflexion plus précise en didactique sur les différentes modalités sensorielles. Pour illustrer ce qui précède, racontons une anecdote. En Italie, la chanson espagnole la cucaracha évoque la fête et reste liée à l'ethno-stéréotype que les Italiens ont de l'Espagne et des pays de langue espagnole. Personne, sauf quelques exceptions, ne s'attarde sur la traduction des mots de cette chanson... il m'est arrivé de la traduire à l'occasion d'une fête, suscitant l'hilarité générale, étant donné que les mots en sont très amusants: la chanson, en effet, narre l'histoire d'une blatte, la «cucaracha» en espagnol, qui n'arrive plus à marcher parce qu'on lui a écrasé une patte. L'anecdote nous permet de réfléchir sur la force évocatrice du signifiant et de comprendre combien l'écoute de ce dernier est un élément essentiel à l'imaginaire et à la relation à l'autre dans le cas d'une langue étrangère. En ce sens, pourquoi ne pas passer par la radio et par la musique pour apprendre avant tout à apprécier et à défendre la différence culturelle? Il nous semble que maintenant plus que jamais, une réflexion sur le potentiel de l'écoute pourrait nous être utile et cela, pourquoi pas, en passant par l'histoire d'un appareil comme la radio, qui, en premier, a su créer des collectifs sociaux bien avant les communautés virtuelles. Nous ne voulons pas nous poser en conservateurs nostalgiques, d'autant plus que notre parcours de vie nous a portée à utiliser plutôt la télévision ou l'ordinateur. Tout simplement nous pensons qu'une réelle prise de conscience des possibilités de l'écoute 
permettrait une meilleure utilisation et exploitation des instruments que nous avons aujourd'hui à disposition dans l'apprentissage des langues étrangères.

\section{BIBLIOGRAPHIE}

\section{Bibliographie}

ARCAINI, Enrico (1959), Corso pratico di lingua francese - Lezioni per la radiotelevisione italiana, Roma, ERI.

------(1973), Progression - Cours radio visuel de français, Roma, ERI.

------(1974-75), Progression - Cours radio visuel de français, Roma, RAI (émission radiophonique).

ELA - De l'éthique en didactique des LE, 109, janvier-mars 1998.

FENATI, Barbara et SCAGLIONI, Alessandra (2002), La radio : modelli, ascolto, programmazione, Roma, Carocci - Le Bussole.

HENDY, David (2002), La radio nell'era globale, Roma, Ed. Riuniti.

MARTINEZ, Pierre (1996), La didactique des langues étrangères, Paris, PUF.

MCLUHAN, Marshall (2002), Gli strumenti del comunicare, Milano, Ed. Net.

MENDUINI, Enrico (2001), Il mondo della radio, Bologna, Il Mulino.

MIRZOEFF, Nicholas (2002), Introduzione alla cultura visiva, Roma, Meltemi.

MONTELEONE, Franco (1995), Storia della Radio e della Televisione in Italia, Venezia, Marsilio Editore.

VARAL, Gilbert (1949), Corso pratico di lingua francese - Lezioni per la radio italiana, Torino, Istituto del Libro Italiano.

-----(1952), Corso pratico di lingua francese - Lezioni per la radio italiana, Torino, ERI.

\section{NOTES}

1. C'est nous qui avons traduit toutes les citations italiennes.

2. Nous tenons à remercier Mme Sisani du bureau de la RAI de Via Cernaia à Turin, qui nous a permis d'accéder au matériel radiophonique.

3. Taxe annuelle d'abonnement.

4. Cf. Monteleone 1995 : 105.

5. Caractéristique de l'Italie de la période fasciste et de l'après-guerre, le «dopolavoro » (aprèstravail) était une association créée pour la récréation des salariés.

6. Cf. Mirzoeff $2002: 13$.

7. Ibidem : 19.

8. Cf. Hendy $2002: 171$. 
9. Pour la différence entre «multimédial » et « multimodal »cf. Lévy, Pierre. 1997. Cyberculture. Paris : O. Jacob.

10. Cf. Monteleone $2001: 256$.

11. Cf. Monteleone $1995: 361$.

12. Cf. Maldonado, Thomas. 1997. Critica della ragione informatica. Milano : Feltrinelli.

13. Cf. Giannini, Jole. 1959. Passaporto - L'inglese alla TV. Torino : ERI.

\section{RÉSUMÉS}

À travers l'analyse d'un corpus constitué des livres-supports aux cours radiophoniques de FLE des années 1949-1973 et des émissions radiophoniques de 1974 en Italie, nous avons reconstruit le parcours des méthodes didactiques en relation à la radio. Nous avons constaté que l'évolution des méthodes se produit en douceur, l'approche précédente contenant les présupposés de la suivante, et que ces changements s'accompagnent de la prise de conscience de l'instrument radiophonique.

Through a survey of the accompanying material of French language radio courses of 1949-1973 and of the programs broadcast in 1974 in Italy, we analyse the smooth evolution of radio teaching methods, with every approach containing the assumptions of the next one, and the changes creating an increasing awareness of radio as a teaching tool.

\section{INDEX}

Mots-clés : Enseignement radiophonique, radiodiffusion scolaire, enseignement du français, Italie, RAI, XXe siècle, Gilbert Varal, Enrico Arcaini

Keywords : Radiophonic teaching, Radio courses, French teaching, Italy, RAI, XXth century, Gilbert Varal, Enrico Arcaini

\section{AUTEUR}

\section{RACHELE RAUS}

Université de Turin 